\title{
Alpha-Fetoprotein, Alpha-Fetoprotein-L3, Protein Induced by Vitamin K Absence, Glypican 3 and Its Combinations for Diagnosis of Hepatocellular Carcinoma
}

\author{
Razvan Cerban ${ }^{1}$, Carmen Ester ${ }^{1}$, Speranta lacob ${ }^{1}$, Mihaela Ghioca ${ }^{1}$, Liliana Paslaru², Radu Dumitru ${ }^{3}$, \\ Mugur Grasu', Georgiana Constantin ${ }^{2}$, Irinel Popescu ${ }^{4}$ and Liliana Gheorghe ${ }^{1}$
}

${ }^{1}$ Centre for Digestive Diseases and Liver Transplantation, Fundeni Clinical Institute, Bucharest, Romania

2Department of Biochemistry, Fundeni Clinical Institute, Bucharest, Romania ${ }^{3}$ Departement of Interventional Radiology, Fundeni Clinical Institute, Bucharest, Romania ${ }^{4}$ Dan Setlacec Centre of General Surgery and Liver Transplantation, Fundeni Clinical Institute, Bucharest, Romania

\section{ABSTRACT}

Introduction: Despite its limitations, alpha-fetoprotein (AFP) is still the most common used serum marker for hepatocellular carcinoma (HCC). Alpha-fetoprotein-L3 (AFP-L3), protein induced by vitamin $\mathrm{K}$ absence (PIVKA-II) and Glypican-3 (GPC-3) have been proposed as complementary biomarkers but their role is still controversial.

Aims and Methods: We prospectively included 101 patients with HCC and 52 control patients with liver cirrhosis with the aim to investigate the diagnostic performance of AFP, AFP-L3 PIVKA-II, and GPC-3 as single markers or in combination for HCC diagnosis. To compare the diagnostic value in distinguishing the presence of HCC from chronic nonmalignant liver disease, receiver operating characteristic (ROC) curves were constructed for each marker and for every combination of markers.

Results: When all biomarkers were individually analyzed, AFP-L3 had the highest area under the curve (AUC) (0.84), followed by AFP (0.79), PIVKA-II (0.75) and GPC-3 (0.73) for HCC diagnosis. The best sensitivity (84.7\%) was for AFP $\mathrm{L} 3$ at a cut-off $>13.5 \mathrm{ng} / \mathrm{mL}$ and the best specificity $(93.9 \%)$ was for AFP at a cut-off $>18.9 \mathrm{ng} / \mathrm{mL}$. For combinations of two biomarkers, the AUC was highest (0.87) for AFP and AFP-L3. The combination of all four biomarkers resulted in a much better sensitivity (88.1\%) and specificity $(93.9 \%)$ than each of the markers individually $(p=0.01)$.

Conclusion: AFP-L3 was the most useful single marker for HCC diagnosis, and the combination of AFP, AFP-L3 and PIVKA-II could maximize the diagnostic performance. Efforts to seek novel combination of biomarkers for HCC should be continued.

Key words: Alpha-fetoprotein, Alpha-fetoprotein-L3, protein induced by vitamin $\mathrm{K}$ absence, Glypican-3, Hepatocellular carcinoma

\section{INTRODUCTION}

Primary liver cancer is one of the main cause of cancer-related death and its incidence has steadily risen over the last 20 years (1). Hepatocellular carcinoma
Corresponding author:

Liliana Gheorghe, MD, PhD

Centre for Digestive Diseases and Liver Transplantation,

Fundeni Clinical Institute, Bucharest Romania

E-mail: drgheorghe@xnet.ro
Abbreviations:

AFP- alpha-fetoprotein; HCC - hepatocellular carcinoma; AFP-L3 - Alpha-fetoprotein-L3; PIVKA-II - protein induced by vitamin K absence; GPC-3 - Glypican-3; ROC - receiver operating characteristic; AUC - area under the curve; LC - liver cirrhosis; 
(HCC) accounts for more than $90 \%$ of all liver cancers, being responsible for more 600000 new cases every year worldwide (2).

The cumulative risk at five years for the HCC development in liver cirrhosis patients has been shown to range between $5 \%$ and $30 \%$ (3). Although there have been advances in diagnosis and treatment modalities, the survival rates of HCC patients has not improved significantly. According to reports, the survival rate at five years of patients with HCC, in the United States remains below $12 \%$ (4).

Diagnosis of HCC in an early stage, followed by surgical resection or liver transplantation offers a good chance for long-term survival of the patients. About $30 \%$ of patients with HCC are diagnosed early enough to undergo a curative treatment.

This is partially due to the limited ultrasound acces for many patients, combined with a poor performance of the currently used tumor marker - $\alpha$-fetoprotein (AFP), which can lead to a delay in diagnosis. Additional sensitive serum markers are definitely needed in order to improve the early diagnosis in individuals at risk for developing HCC.

Serum AFP, considered by far the most extensivelyused HCC biomarker, has a relatively low diagnostic sensitivity, due to the fact that its levels are elevated not only in patients with HCC but also and in those with chronic liver disorders. In subjects diagnosed with chronic hepatitis $\mathrm{C}$, the most prevalent type of viral hepatitis in Romania, AFP is often elevated in the absence of HCC, but has a normal value in as many as $50 \%$ of patients with $\mathrm{HCC}(5)$.

Study of the chemical structure of AFP revealed that there are different sugar components of the bonds that determine their binding capacity to Lens culinaris agglutinin (6). Lens culinaris agglutininreactive AFP (AFP-L3) is the major glycoform in the serum of patients with $\mathrm{HCC}$ and is more specific than AFP (7). The AFP-L3 isoform has also been shown to be associated with more aggressive HCCs, and to predict a worse outcome (8).

Protein induced by vitamin $\mathrm{K}$ absence or antagonistII (PIVKA-II) is an abnormal prothrombin that can be detected at higher levels in the serum of patients diagnosed with HCC. Since the initial report by Liebman et al. (9), PIVKA-II has been established as a marker with high specificity for HCC and a predictor of the prognosis of patients with $\operatorname{HCC}(6,10,11)$. Several studies have shown that performing a combined measurement of PIVKA-II and AFP resulted in a sensitivity ranging from $50 \%$ to $90 \%$, and a specificity ranging from more $50 \%$ to almost $99 \%$ for early HCC diagnosis, these values were superior to those for either biomarker alone $(12,13)$.

For early HCC, measurement of both tumor markers is recommended, since PIVKA $\|$ is a more specific marker compared with AFP (14). A high PIVKA-II level implies a poor prognosis, and a slight increase in its concentration after therapy could suggest recurrence.

Glypican-3 (GPC-3) is a heparin sulfate proteoglycan that was evaluated as a biomarker for the HCC diagnosis, based on the fact that it was detected in liver cancer cells and not in benign liver tissues (15). It was reported that serum levels of GPC-3 above $300 \mathrm{ng} / \mathrm{L}$ had a sensitivity of $47.0 \%$ and specificity of more $90 \%$ for diagnosing HCC (16). In addition, the diagnostic accuracy of GPC-3 was shown to be increased when tested in combination with AFP (17).

The objective of the current study was to determine the diagnostic performance of the four biomarkers for HCC detection by analyzing the sensitivity and specificity of each single biomarker and their combination in a cohort of HCC patients and a control group of patients with liver cirrhosis (LC).

\section{MATERIALS AND METHODS}

\section{Patients}

A total of 153 patients (101 HCC and 52 LC) were enrolled at Fundeni Clinical Institute from March 2016 to December 2018.

All patients were newly diagnosed with $\mathrm{HCC}$, without any previous HCC treatment and none had extrahepatic malignancy at the time of their diagnosis. Plasma samples were obtained from all the patients included in the study and from patients that underwent resection or liver transplant also tissue samples were collected for future studies. HCC was diagnosed based on typical imaging findings as defined by the EASL guidelines (18). Barcelona Clinic Liver Cancer (BCLC) classification system was used for HCC staging (5).

The diagnosis of LC was established using one or more findings of portal hypertension: 1) liver with a cirrhotic aspect associated with splenomegaly on imaging examinations (ultrasonography, computed tomography or magnetic resonance), 2) presence of esophageal or gastric varices on endoscopy 3) thrombocytopenia (platelet $<100,000 / \mathrm{mm}$ ), 4) presence of ascites, and 5) evidence of hepatic encephalopathy. All patients with LC included in the control group underwent imaging examination to exclude the presence of HCC.

All clinical and laboratory data were prospectively collected for all the patients and included liver trans- 
aminases, bilirubin, albumin, creatinine, platelet count, INR hepatitis $B$ and $C$ serologies.

This study was conducted with approval from the Ethics Committee of the Fundeni Clinical Institute. An informed consent in written was obtained from all included participants.

\section{Sample storage and assays}

A peripheral blood sample was collected from each patient at the time of the initial diagnosis for the HCC group or during the clinic visit for the control group. Until measurement, serum aliquots were stored at $35^{\circ} \mathrm{C}$.

Serum AFP, AFP L3, PIVKA-II and GPC-3 levels were measured in the same samples. Serum AFP and PIVKA-II were determined by use of automated chemiluminescent microparticle immunoassays (ARCHITECT AFP 3P36; ARCHITECT PIVKA-II 2P4) according to the manufacturer's instructions, utilizing the Abbott $^{\mathrm{TM}}$ Architect System 1000 analyser.

AFP-L3 and GPC-3 were measured using commercially available enzyme-linked immunosorbent assay (ELISA) in accordance with the instructions of the manufacturer (AFP L3 - Catalog No: E-EL-H0289; GPC-3 Catalog No: E-EL-H1712, ElabScience Co. Wuhan China). The measurement range was 0.63 to $40 \mathrm{ng} / \mathrm{mL}$ for AFP-L3 and 160 to $10000 \mathrm{pg} / \mathrm{mL}$ for GPC-3.

Adequate standard curves were calculated for each ELISA plate that was used. In the cases where AFP level of a sample was $>2000 \mathrm{ng} / \mathrm{mL}$ or the PIVKA-II level was $>30,000 \mathrm{mAU} / \mathrm{mL}$, the original sample was diluted according to the instructions of the manufacturer. All tests were performed at the Biochemistry Department of Fundeni Clinical Institute by the same group of experienced laboratory technicians, who were not informed on clinical data of any patient. Refreezing of thawed plasma samples was not performed.

\section{Statistical analysis}

The data are presented as mean \pm SD and median (range) or as relative frequencies (\%) when appropriate. We used Mann-Whitney test and the $\chi^{2}$ test to perform the comparison of continuous and dichotomous variables between the two groups. For correlations between tumor markers, Pearson's correlation coefficient was calculated.

For all of the tests of significance a $p$ value lower than 0.05 was regarded as statistically significant.

To compare the diagnostic performance of each marker and every combination of markers, receiver operating characteristics (ROC) curves were constructed. We determined the optimal cut-off value by calculating the Youden index for each ROC curve. Differences between the area under the ROC curve (AUC) of each biomarker for distinguishing between HCC and LC patients and their 95\% confidence intervals (Cl) were calculated. We considered a positive combination if any biomarker in the combination was positive.

Statistical analyses were performed using Medcalc ver. 13.3.3.0 software (Medcalc Software).

\section{RESULTS}

Both clinical and biological characteristics of the study population $(n=153)$ are presented in table 1 . There was a higher proportion of males $(p=0.04)$ and the subjects tended to be older $(p=0.03)$ in the group of patients diagnosed with HCC, compared with the LC control group. In our study all patients with HCC had cirrhosis as their underlying liver disease. There was no difference in the serum levels of alanine aminotransferase (ALT) and of total bilirubin between the two groups of patients.

The most common etiology in both study groups was HCV infection, followed by hepatitis B infection. No significant differences according to Child-Pugh class was observed between the two groups of patients.

Considering the fact that serum levels of biomarkers were sometimes extremely high, we decided to measure median and interquartile range (IQR) values to allow more accurate comparisons between values and avoid misleading mean values. Median serum values for all four biomarkers were observed to be significantly higher in subjects with HCC than in the LC group.

Each studied biomarker was observed to have a different serum level distribution and range. We performed an analysis of the correlation coefficients to identify statistically significant correlations between the markers. The correlation coefficient for AFP and PIVKAII was 0.107 (fig. 1), whereas that for AFP and GPC-3 was 0.102 (fig. 2). We demonstrated a good correlation between AFP and AFP-L3, with a coefficient of 0.51 (fig. 3).

\section{Biomarkers performance evaluation}

Receiver operator characteristic curves (ROC) for HCC diagnosis using total AFP, AFP-L3, PIVKA-II and GPC-3 are shown in fig. 4.

For the 4 biomarkers individually, in distinguishing between HCC and chronic liver disease, AFP L3 showed the highest area under the ROC curve (AUC) (0.842, 
Table 1 - Characteristics of the patients

\begin{tabular}{|c|c|c|c|}
\hline & HCC $(n=101)$ & LC $(n=52)$ & $p$ value \\
\hline Age, mean years & $60,3 \pm 8,52$ & $59,4 \pm 8,4$ & $p=0.03$ \\
\hline Gender, n (male) & $67(66.3 \%)$ & $27(51.9 \%)$ & $p=0.04$ \\
\hline \multicolumn{4}{|l|}{ Ėtiology } \\
\hline $\mathrm{HCV}$ & $63(62.4 \%)$ & $30(57.7 \%)$ & $p=0.86$ \\
\hline $\mathrm{HBV}$ & $24(23.7 \%)$ & $13(25 \%)$ & \\
\hline Alcohol & $12(11.9 \%)$ & $7(13.5 \%)$ & \\
\hline Others & $2(1.9 \%)$ & $2(3.8 \%)$ & \\
\hline \multicolumn{4}{|l|}{ Child-Pugh class } \\
\hline$A$ & $78(77.2 \%)$ & $30(57.7 \%)$ & $p=0.24$ \\
\hline $\mathrm{B}$ & $17(16.8 \%)$ & $12(23.1 \%)$ & \\
\hline $\mathrm{C}$ & $6(5.9 \%)$ & $10(19.3 \%)$ & \\
\hline Platelet, $\times 10^{3} / \mathrm{L}$ & $99(74.50-139.50)$ & $81(67.0-133.0)$ & $p=0.36$ \\
\hline Ailbumin, $g / L$ & $3.7(3.08-4.0)$ & $3(2.7-3.5)$ & $p=0.01$ \\
\hline Bilirubin, mg/dL & $1(1-1.75)$ & $1.2(1.10-1.85)$ & $p=0.23$ \\
\hline ALT, UI/L & $54(37-109)$ & $49(32-115)$ & $p=0.65$ \\
\hline INRR & $1.25(1.00-1.70)$ & $1.20(1.05-1.45)$ & $p=0.93$ \\
\hline MELD score & $10(8-12)$ & $11(8.25-15.00)$ & $p=0.18$ \\
\hline AFP, $n g / m L$ & $18(6.63-104.56)$ & $4.75(3.21-6.70)$ & $p<0.0001$ \\
\hline ÄFP L3, ng/mL & $23.7(16.53-40.00)$ & $12(6.97-14.35)$ & $p<0.0001$ \\
\hline PIVKA II, $\mathrm{mUA} / \mathrm{mL}$ & $188.4(81.36-723.21)$ & $63(34.73-160.03)$ & $p<0.0001$ \\
\hline GPC-3, pg/ml & $601(475.75-1989.00)$ & $415(250.00-527.75)$ & $\mathrm{p}<0.0001$ \\
\hline
\end{tabular}

Data are expressed as mean \pm SD, or median (interquartile range) for quantitative variables and as number or cases (percentage) for qualitative variables.

$L C=$ liver cirrhosis; $H C C=$ hepatocellular carcinoma; $H B V=$ hepatitis $B$ virus; $H C V=$ hepatitis $C$ virus; $A L T=$ alanine

aminotransferase; INR =international normalized ratio; $M E L D=$ model for end-stage liver disease; $A F P=$ alpha-fetoprotein; PIVKA-II= protein induced by vitamin $\mathrm{K}$ absence; GPC-3=glypican-3

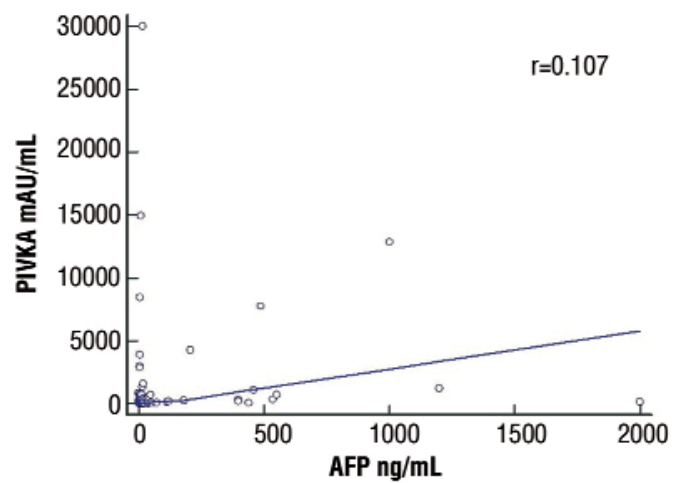

Figure 1 - Linear correlation between AFP and PIVKA II

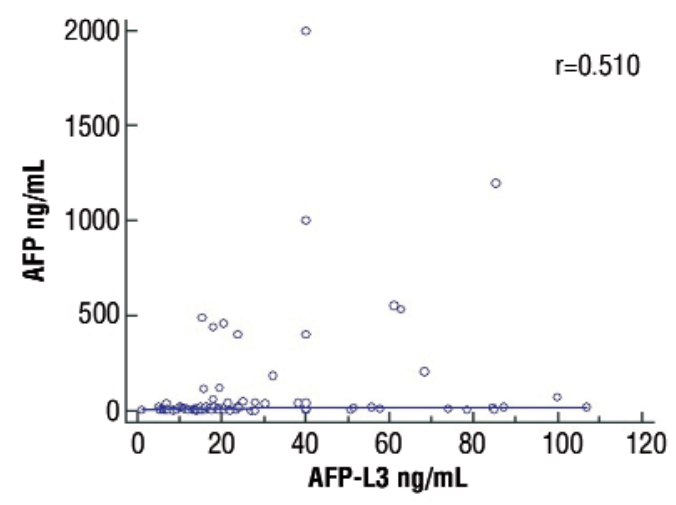

Figure 3 - Linear correlation between AFP and AFP L3

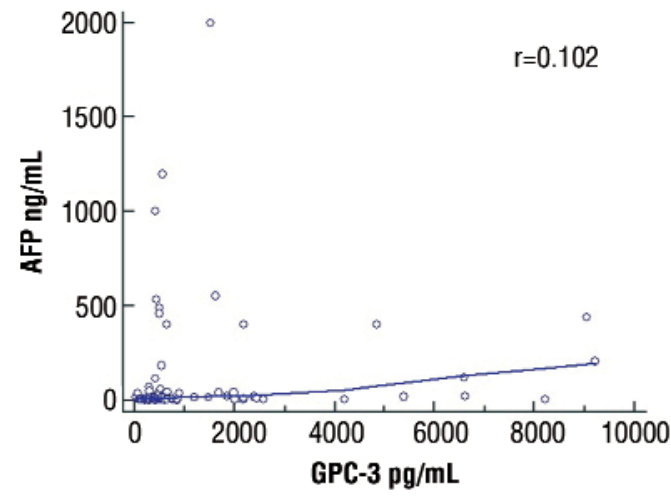

Figure 2 - Linear correlation between AFP and GPC-3

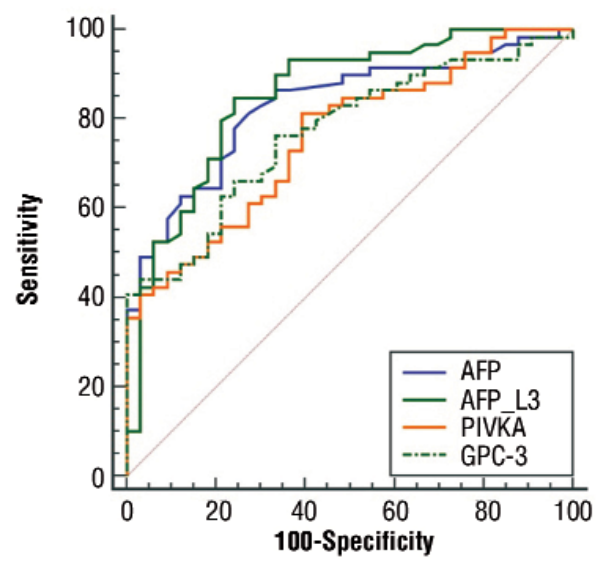

Figure 4 - ROC curves for the diagnosis of HCC using AFP, AFP-L3, PIVKA II and GPC-3 
95\% confidence interval $[\mathrm{Cl}]$ 0.763-0.911), followed by AFP $(0.798,95 \%$ confidence interval $[\mathrm{Cl}]$ 0.738-0,881). As a single marker GPC-3 had the lowest AUC (0.730, $95 \%$ confidence interval $[\mathrm{Cl}] 0,670$ to 0,831 ) (table 2). There was no significant differences of the AUC among the individual biomarkers.

The optimal cut-offs that resulted from the Youden index for AFP, AFP-L3, PIVKA-II and GPC-3 were 18.9 $\mathrm{ng} / \mathrm{mL}, 13.5 \mathrm{ng} / \mathrm{mL}, 63 \mathrm{mAU} / \mathrm{mL}$, and $466 \mathrm{pg} / \mathrm{mL}$ respectively. Of the 101 serum samples from patients diagnosed with HCC, 50 samples (49.5\%) were positive for AFP, 78 samples (77.2\%) were positive for AFP-L3, 48 samples (47.5\%) were positive for PIVKA-II and 46 samples (45.5\%) were positive for GPC-3. As diagnostic markers for HCC, AFP > $18.9 \mathrm{ng} / \mathrm{mL}$ was the most specific (92\%) but with a lower sensitivity than AFP L3 (52.5\% vs. 87.7\%).

When we studied the diagnostic utility of the combination of two biomarkers, AFP > $18.9 \mathrm{ng} / \mathrm{mL}$ combined with AFP L3 > $13.5 \mathrm{ng} / \mathrm{mL}$ showed the highest area under the ROC curve (AUC) $(0.872,95 \%$ confidence interval [Cl] 0,799 to 0.930), an increased sensitivity (74.5\%), and a similar specificity as compared to AFP alone. The combination with the greatest specificity using two biomarkers was AFP-L3 >13.5 $\mathrm{ng} / \mathrm{mL}$ combined with PIVKA-II $>63 \mathrm{mAU} / \mathrm{mL}$ (specificity of $96.9 \%$, sensitivity of $71.2 \%$ ) (table 2 ).

As to triple biomarker combinations, the AFP $>18.9$ $\mathrm{ng} / \mathrm{mL}$ and AFP L3> $13.5 \mathrm{ng} / \mathrm{mL}$ and PIVKA-II > 466 $\mathrm{mAU} / \mathrm{mL}$ combination showed the best sensitivity $(84.7 \%)$ with a specificity of 90.9 lower that of the combination of AFP L3 and PIVKA-II. The combination of all four biomarkers (AFP, AFP L3, PIVKA-II and GPC-3), improved the sensitivy (83.1\%) compared and the best two-marker combination but with a lower specificity (93.9\%).

\section{DISCUSSION}

Early HCC diagnosis is essential to ensure that treatments with a curative intent can be carried out to improve the long-term prognosis and survival of patients (19).

The latest Western guidelines no longer recommends AFP measurement for evaluation of patients for the presence of HCC, because of its limited value in HCC detection, due to the sensitivity of about $60 \%$ at a cut-off value of $20 \mathrm{ng} / \mathrm{mL}(18,20,21,22)$ and low specificity (23). To overcome this, investigators have examined other biological molecules in an attempt to

Table 2 - Diagnostic value of AFP, AFP-L3, PIVKA-II, GPC-3 and their combinations in discriminating HCC from LC

\begin{tabular}{|c|c|c|c|c|c|c|}
\hline \multirow[t]{2}{*}{ Number of markers } & \multirow[t]{2}{*}{ Combination } & \multicolumn{5}{|c|}{ Total subjects ( $n=153$ ) } \\
\hline & & Sn & $S p$ & PPV & NPV & AUC \\
\hline \multirow[t]{4}{*}{ Single } & AFP & 52.54 & 93.94 & 93.9 & 52.5 & 0.798 \\
\hline & PIVKA-II & 81.36 & 60.61 & 78.7 & 64.5 & 0.752 \\
\hline & AFP L3 & 84.75 & 75.76 & 86.2 & 73.5 & 0.842 \\
\hline & GPC & 76.27 & 66.67 & 80.4 & 61.1 & 0.730 \\
\hline \multirow[t]{6}{*}{ Double } & AFP + PIVKA & 74.58 & 87.5 & 91.7 & 65.1 & 0.852 \\
\hline & AFP +AFP L3 & 74.58 & 92.94 & 95.7 & 67.4 & 0.872 \\
\hline & AFP + GPC3 & 74.58 & 90.91 & 93.6 & 66.7 & 0.85 \\
\hline & PIVKA + AFP L3 & 71.19 & 96.97 & 97.7 & 65.3 & 0.859 \\
\hline & PIVKA+ GPC3 & 74.58 & 91.93 & 95.7 & 67.4 & 0.866 \\
\hline & AFP L3 + GPC 3 & 79.66 & 87.88 & 92,2 & 70.7 & 0.862 \\
\hline \multirow[t]{4}{*}{ Triple } & AFP + PIVKA + AFP L3 & 84.75 & 90.91 & 94.3 & 76.9 & 0.916 \\
\hline & AFP + PIVKA + GPC3 & 86.44 & 87.88 & 92.7 & 78.4 & 0.905 \\
\hline & $\mathrm{AFP}+\mathrm{AFP} \mathrm{L} 3+\mathrm{GPC} 3$ & 76.27 & 96.97 & 97.8 & 69.6 & 0.896 \\
\hline & PIVKA + AFP L3 + GPC3 & 77.97 & 96,97 & 97.9 & 71.1 & 0.90 \\
\hline Quadruple & AFP+PIVKA+AFP L3+GPC 3 & 88.14 & 93.94 & 96.3 & 81.6 & 0.917 \\
\hline
\end{tabular}

Cut-off values for each biomarker were: AFP >18.9 ng/mL, AFP L3 >13.5 ng/ml, PIVKA-II > 63 mAU/ml, $\mathrm{GPC}>466 \mathrm{pg} / \mathrm{mL}$

LC = liver cirrhosis; HCC = hepatocellular carcinoma, AFP = alpha-fetoprotein; PIVKA-II = protein induced by vitamin $\mathrm{K}$ absence; $\mathrm{GPC}-3$ = glypican- $3, \mathrm{Sn}=$ sensitivity; $\mathrm{Sp}=$ specificity; $\mathrm{PPV}=$ positive predictive value; $\mathrm{NPV}=$ negative predictive value; $\mathrm{AUC}=$ area under the curve 
find better diagnostic markers for HCC.

It was demonstrated in previous studies that PIVKAII could be useful for the early diagnosis of small HCC tumors (24). In addition, several papers most of them from Asia have shown that combining AFP-L3 with PIVKA-II could improve the rate of HCC detection $(8,25$, 26). Lim et al showed that combining AFP, AFP-L3, and PIVKA-II can improve the diagnostic performance for HCC among subjects with LC in comparison with each biomarker used individually (27).

The aim of the current study is to evaluate if AFP L3, PIVKA-II and GPC-3 could be useful as di-agnostic biomarkers for HCC in comparison to AFP in for the first time in Romanian patients. In order to achieve this, we performed a case-control study with the aim to identify new valuable diagnostic biomarkers that can be used in daily practice.

Chronic hepatitis $C$ is considered to be the major cause of cirrhosis and HCC in Western countries (28). In our study, chronic HCV hepatitis was the most frequent cause in both HCC and LC control group. The results from our study are therefore likely to be representative of the total Romanian population.

Considering the fact that HCC development is a major cause of death in patients with LC (29) and most HCC patients have underlying LC, we decided to include in our study only LC patients as a control group.

We have evaluated the utility of AFP, AFP-L3, PIVKA-II and GPC-3 for HCC diagnosis, by performing a direct comparison individually and in combination, and found that AFP-L3 was the best individual marker for differentiating between HCC and LC (sensitivity 84.7\%, specificity $75.7 \%$, AUC $0.842,95 \% \mathrm{Cl} 0,763-0,918$ ). There was no statistical significant difference between AFP-L3 and the other biomarkers.

PIVKA-II is a precursor of prothrombin and its synthesis is induced by vitamin $\mathrm{K}$ deficiency. Tumor liver cells have lower levels of vitamin $\mathrm{K}$ than normal cells and this fact explains for the extremely low levels of PIVKA-II in healthy individuals. Additionally PIVKA-II is elevated not only in HCC subjects, but also in chronic hepatitis or liver cirrhosis patients, which limits its diagnostic accuracy (30). In our study we found that as a single marker PIVKA II had a sensitivity of $81.3 \%$ compared to $52.5 \%$ for AFP for diagnosis of HCC. Several studies have demonstrated that PIVKA-II is detected in AFP-seronegative HCC patients and offers better sensitivity than AFP for HCC diagnosis, which is consistent with our findings $(31,32)$.

Since it was initially reported to be positive in up to $50 \%$ of subjects with HCC, but undetectable in controls without liver disease, GPC-3 has been investi- gated as a diagnostic biomarker for HCC (33). Althought initially it was considered that GPC-3 could replace or complement AFP, later studies have yielded variable results (34). In our study the sensitivity, specificity, and AUC of GPC-3 were comparable to previous results and the cut-off value $(466 \mathrm{pg} / \mathrm{mL})$ determined by ROC analysis was different than other reported values, which range widely from $3.9 \mathrm{pg} / \mathrm{mL}$ to $300 \mathrm{ng} / \mathrm{mL}$ (35). In our analysis GPC-3 had the lowest AUC of all studied biomarkers $(0.730,95 \% \mathrm{Cl})$ and as a single marker, failed to be useful for HCC diagnostis compared to AFP.

Among all combinations of two biomarkers, AFP $>18.9 \mathrm{ng} / \mathrm{mL}$ and AFP-L3 $>13.5 \mathrm{ng} / \mathrm{mL}$ had the highest AUC $(0.872,95 \% \mathrm{Cl})$, with a sensitivity of $74.58 \%$ and a specificity of $92.94 \%$.

Triple panels did improve the diagnostic performance compared to the best results achieved from using two biomarkers. Performing the combined measurement of AFP, AFP L3 and PIVKA-II resulted in the best AUC, (sensitivity $84.75 \%$, specificity $90.91 \%$, AUC $0.916,95 \% \mathrm{Cl} 0.852-0.940$ ). Adding GPC-3 to the combination only marginally increased the sensitivity (88.14\%), specificity $(93.94 \%)$ and the AUC $(0.917,95 \%$ $\mathrm{Cl}$ ) of the panel but with a cost increase.

A diagnostic biomarker for HCC should be highly sensitive, should differentiate HCC from other liver diseases, and should be detected in the plasma at high levels even in early stages. In our study, serum AFP, AFP L3, PIVKA-II and GPC-3 failed to achieve these standards as single markers, but their combination greatly improved HCC detection rate.

One limitation of the present study is that all the samples were collected at single center, and so the results that we obtined need external validation. However, repeated experiments for internal validation were strictly performed and in all of these cases, we used new aliquots of stored samples.

Another limitation of this study is that we used only one commercially avail-able ELISA assay for each of the studied biomarkers. The enrolled patients with HCC were heterogeneous in regard to liver disease etiology, tumor stage and underlying liver function, which reflects real-world practice, but this fact may lead to variability. Considering the fact that clinical utility of a biomarker panel should be considered after taking into account cost-effectiveness, further analyses are necesary to determine the proper number and combination of biomarkers.

Future studies should be performed in order to clarify several issues. By using larger sample sizes, more accurate estimates for the sensitivity and specificity of the panel will be established. Since levels of the 
markers are much higher in HCC patients than in nonHCC patients and healthy individuals, different cut-off levels can be tried to further improve the specificity.

\section{CONCLUSION}

In conclusion, AFP and AFP-L3 seems to be the most valuable two marker combination for the diagnosis of HCC, as shown by comparative analyses of AFP, AFP-L3, PIVKA-II, and GPC-3, especially in a HCV-predominant HCC population. Persistent inconsistency in plasma GPC-3 levels questions its utility as a diagnostic biomarker for HCC compared to AFP. PIVKA-II was demonstrated to be a useful complementary marker if it is associated with AFP and AFP L3. Prospective studies to find the most efficient and cost-effective biomarker panel are needed.

\section{Conflict of interest}

All author declare that they have no conflict of interest.

\section{REFERANCES}

1. International Agency for Research on Cancer: Number of cancer deaths: World Health Organization, mortality database. (accessed on 02/01/2019).

2. Ferlay J, Shin HR, Bray F, Forman D, Mathers C, Parkin DM. GLOBOCAN 2008 v2.0, Cancer Incidence and Mortality Worldwide: IARC Cancer Base No. 10. Lyon, France: International Agency for Research on Cancer; 2010.

3. Fattovich G, Stroffolini T, Zagni I, Donato F: Hepatocellular carcinoma in cirrhosis: incidence and risk factors. Gastroenterology 2004;127:S35-S50.

4. El-Serag HB: Hepatocellular carcinoma. N Engl J Med 2011, 365:1118-1127.

5. Llovet JM, Di Bisceglie AM, Bruix J, Kramer BS, Lencioni R, Zhu $A X$, et al. Design and endpoints of clinical trials in hepatocellular carcinoma. J Natl Cancer Inst. 2008;100(10):698-711.

6. Zhou L, Liu J, Luo F. Serum tumor markers for detection of hepatocellular carcinoma. World J Gastroenterol 2006; 12: 1175-81.

7. Malaguarnera G, Giordano M, Paladina I, Berretta M, Cappellani A, Malaguarnera M. Serum markers of hepatocellular carcinoma. Dig Dis Sci 2010;55:2744-55.

8. Hayashi K, Kumada T, Nakano S, Takeda I, Sugiyama K, Kiriyama S, et al. Usefulness of measurement of Lens culinaris agglutininreactive fraction of alphafetoprotein as a marker of prognosis and recurrence of small hepatocellular carcinoma. Am J Gastroenterol 1999:94: 3028-33.

9. Liebman HA, Furie BC, Tong MJ, Blanchard RA, Lo KJ, Lee SD, et al. Des-gamma-carboxy (abnormal) prothrombin as a serum marker of primary hepatocellular carcinoma. N Engl J Med 1984;310:1427-31.

10. Koike $Y$, Shiratori $Y$, Sato S, Obi S, Teratani T, Imamura M, et al. Des-gamma-carboxy prothrombin as a useful predisposing factor for the development of portal venous invasion in patients with hepatocellular carcinoma: a prospective analysis of 227 patients. Cancer 2001;91:561-9.

11. Imamura H, Matsuyama $\mathrm{Y}$, Miyagawa $\mathrm{Y}$, Ishida $\mathrm{K}$, Shimada $\mathrm{R}$, Miyagawa $S$, et al. Prognostic significance of anatomical resection and des-gamma-carboxy prothrombin in patients with hepatocellular carcinoma. Br J Surg 1999;86:1032-8.
12. Shimauchi $\mathrm{Y}$, Tanaka $\mathrm{M}$, Kuromatsu $\mathrm{R}$, Ogata $\mathrm{R}$, Tateishi $\mathrm{Y}$, Itano $\mathrm{S}$, et al. A simultaneous monitoring of Lens culinaris agglutinin A-reactive alpha-fetoprotein and desgamma- carboxy prothrombin as an early diagnosis of hepatocellular carcinoma in the follow-up of cirrhotic patients. Oncol Rep 2000;7:249-56.

13. Durazo FA, Blatt LM, Corey WG, Lin JH, Han S, Saab S, et al. Des-gamma-carboxyprothrombin, alpha-fetoprotein and AFP-L3 in patients with chronic hepatitis, cirrhosis and hepatocellular carcinoma. J Gastroenterol Hepatol 2008;23:1541-8.

14. Marrero JA, Su GL, Wei W, Emick D, Conjeevaram HS, Fontana RJ, et al. Des-gamma carboxyprothrombin can differentiate hepatocellular carcinoma from nonmalignant chronic liver disease in american patients. Hepatology. 2003;37(5):1114-21.

15. Shafizadeh N, Ferrell LD, Kakar S: Utility and limitations of glypican-3 expression for the diagnosis of hepatocellular carcinoma at both ends of the differentiation spectrum. Mod Pathol 2008; 21:1011-1018.

16. Liu H, Li P, Zhai Y, Qu CF, Zhang LJ, Tan YF, et al. Diagnostic value of glypican-3 in serum and liver for primary hepatocellular carcinoma. World J Gastroenterol 2010, 16:4410-4415.

17. Qiao SS, Cui ZQ, Gong L, Han H, Chen PC, Guo LM, et al. Simultaneous measurements of serum AFP, GPC-3 and HCCR for diagnosing hepatocellular carcinoma. Hepatogastroenterology 2011;58:1718-1724.

18. European Association for the Study of the Liver, European Organisation for Research Treatment of Cancer. EASL-EORTC clinical practice guidelines: management of hepatocellular carcinoma. J Hepatol 2012; 56(4):908-943.

19. Llovet JM, Burroughs A, Bruix J. Hepatocellular carcinoma. Lancet 2003;362:1907-17.

20. Bruix J, Sherman M, American Association for the Study of Liver Disease. Management of hepatocellular carcinoma: an update. Hepatology 2011;53(3):1020-1022.

21. Marrero JA, Feng Z, Wang Y, Nguyen MH, Befeler AS, Roberts LR, et al. Alpha-fetoprotein, desgamma carboxyprothrombin, and lectin-bound alpha-fetoprotein in early hepatocellular carcinoma. Gastroenterology 2009; 137(1):110-118.

22. Adachi Y, Tsuchihashi J, Shiraishi N, Yasuda K, Etoh T, Kitano S. AFP-producing gastric carcinoma: multivariate analysis of prognostic factors in 270 patients. Oncology 2003; 65(2):95-101.

23. Chaiteerakij R, Addissie BD, Roberts LR. Update on biomarkers of hepatocellular carcinoma. Clin Gastroenterol Hepatol 2015; 13(2): 237-245.

24. Mita $Y$, Aoyagi $Y$, Yanagi M, Suda T, Suzuki $Y$, Asakura $H$, et al. The usefulness of determining desgamma- carboxy prothrombin by sensitive enzyme immunoassay in the early diagnosis of patients with hepatocellular carcinoma. Cancer 1998;82:1643-8

25. Choi JY, Jung SW, Kim HY, Kim M, Kim Y, Kim DG, et al. Diagnostic value of AFP-L3 andPIVKA-II in hepatocellular carcinoma according to total-AFP. World J Gastroenterol 2013;19:339-46.

26. Hadziyannis E, Sialevris K, Georgiou A, Koskinas J. Analysis of serum alphafetoprotein-L3\% and des-gamma carboxyprothrombin markers in cases with misleading hepatocellular carcinoma total alpha-fetoprotein levels. Oncol Rep 2013;29:835-9.

27. Lim TS, Kim do Y, Han KH, Kim HS, Shin SH, Jung KS, et al. Combined use of AFP, PIVKA-II, and AFP-L3 as tumor markers enhances diagnostic accuracy for hepatocellular carcinoma in cirrhotic patients. Scand J Gastroenterol 2016;51: 344-53.

28. Kim MN, Kim BK, Han KH. Hepatocellular carcinoma in patients with chronic hepatitis $\mathrm{C}$ virus infection in the Asia-Pacific region. J Gastroenterol 2013;48:681-8.

29. El-Serag HB, Davila JA, Petersen NJ, McGlynn KA. The continuing increase in the incidence of hepatocellular carcinoma in the United States: an update. Ann Intern Med 2003;139:817-23.

30. Bertino G, Ardiri AM, Calvagno GS, Bertino N, Boemi PM. Prognostic and diagnostic value of des-g-carboxy prothrombin in liver cancer. Drug News Perspect 2010; 23(8): 498-508.

31. Marrero JA, Su GL, Wei W, Emick D, Conjeevaram HS, Fontana RJ, et al. Des-gamma carboxyprothrombin can differentiate hepatocellular carcinoma from nonmalignant chronic liver disease in American patients. Hepatology 2003; 37(5): 1114-1121. 
32. Ismail S, Mayah W, Battia HE, Gaballah H, Jiman-Fatani A, Hamouda $\mathrm{H}$, et al. Plasma nuclear factor kappa $\mathrm{B}$ and serum peroxiredoxin 3 in early diagnosis of hepatocellular carcinoma. Asian Pac $\mathrm{J}$ Cancer Prev 2015; 16(4):1657-1663.

33. Capurro M, Wanless IR, Sherman M, Deboer G, Shi W, Miyoshi E, et al. Glypican-3: a novel serum and histochemical marker for hepatocellular carcinoma. Gastroenterology 2003;125:89-97.
34. Jeon Y, Jang ES, Choi YS, Kim JW, Jeong SH. Glypican-3 level assessed by the enzyme-linked immunosorbent assay is inferior to alpha-fetoprotein level for hepatocellular carcinoma diagnosis. Clin Mol Hepatol. 2016;22(3):359-365.

35. Jia X, Liu J, Gao Y, Huang Y, Du Z. Diagnosis accuracy of serum glypican-3 in patients with hepatocellular carcinoma: a systematic review with meta-analysis. Arch Med Res 2014:45:580-588. 